\title{
El (des)empleo en la profesión de periodista en tiempos de pandemia
}

\author{
(Un) employment in the journalism profession in times of pandemics
}

\author{
Daniel Rodrigo Cano \\ Centro Universitario San Isidoro | Calle Leonardo da Vinci, 17, b, 41092 Sevilla | España | \\ https://orcid.org/0000-0003-2753-5470 | drodrigo@centrosanisidoro.es

\section{Noelia Ruiz-Alba} \\ Universidad de Sevilla | Calle Américo Vespucio, s/n, 41092, Isla de la Cartuja, Sevilla | España | \\ https://orcid.org/0000-0001-9438-5519 | noeliaruizalba@gmail.com
}

Fechas | Recepción: 21/03/2021 | Aceptación: 30/03/2021

Resumen

Durante la pandemia de la COVID-19 se provocó la paralización de la economía durante muchos meses y durante el confinamiento, sin embargo, la comunicación era declarada como actividad esencial. Para conocer el posible impacto de esta situación y poder adelantar un escenario futuro de la profesión de periodista se ha realizado un análisis del paro registrado por comunidades autónomas desde 2008 hasta 2020. Los resultados muestran que el desempleo en la profesión ha visto un incremento en 2020 un 25\% con respecto al mismo mes del año anterior. Además, si se repite la tendencia de la anterior crisis de desempleo en España desde 2008 a 2013 aún se van a perder numerosos puestos de trabajo de periodistas. Por otra parte, los datos de la Asociación de la Prensa de Madrid entre los años 2008 y 2020 indican que, son las mujeres periodistas las que más desempleo mantienen y en concreto las comunidades autónomas de Madrid, Andalucía y Catalunya. Junto con el retroceso en la profesión de periodista emergen las noticias falsas y los bulos lo que plantea la paradoja de necesitar más periodistas, más especialización, más local y con la exigencia de formar una ciudadanía que requiere conocer la verdad especialmente en tiempos de emergencia sanitaria.

Palabras clave: periodista, periodismo, desempleo, precariedad, crisis, pandemia

\section{Abstract}

During the Covid-19 pandemic, the economy was paralysed for many months and during the confinement, however, communication was declared an essential activity. In order to understand the possible impact of this situation and to be able to anticipate a future scenario for the journalism profession, an analysis of registered unemployment by autonomous community from 2008 to 2020 has been carried out. The results show that unemployment in the profession has seen a $25 \%$ increase in 2020 compared to the same month of the previous year. Moreover, if the trend of the previous unemployment crisis in Spain from 2008 to 2013 is repeated, many journalists' jobs will still be lost. On the other hand, data from the Madrid Press Association between 2008 and 2020 indicate that women journalists are the most unemployed, particularly in the autonomous communities of Madrid, Andalusia and Catalonia. Along with the decline in the profession of journalism, fake news and hoaxes are emerging, which raises the paradox of needing more journalists, more specialization, more local and with the requirement to train a citizenry that needs to know the truth, especially in times of health emergencies.

Keywords: journalist, journalism, unemployment, precariousness, crisis, pandemic 


\section{INTRODUCCIÓN}

El periodismo es un oficio precarizado (Soengas et al. 2014) y desde 2020, expuesto al Covid-19 (Grecko, 2021). El periodista debe salir a la calle para contar lo que ocurre, asiste a menudo a convocatorias y ruedas de prensa y necesita estar en contacto con las fuentes. Muchas de estas rutinas se han trasladado también a la esfera digital, aunque muchas otras deben realizarse ineludiblemente de forma presencial, como es en el caso de algunos trabajadores de televisión. Por ello, los periodistas conforman un colectivo de profesionales expuesto al coronavirus.

Se trata de una profesión que, según la Unesco (2007), desempeña diferentes funciones sociales, como fuente de información, análisis y comentario de la actualidad, para ello es necesario que los y las periodistas informen a la sociedad, se interroguen sobre el modo de proceder del poder, favorece el debate democrático y, de esta forma, contribuir al desarrollo político, económico, social y cultural.

Sin embargo, la percepción social de los españoles y españolas respecto de la profesión de periodista no es muy buena, así mientras médico o docente en los diferentes niveles educativos tienen valoraciones muy buenas, por encima del 30\% mantienen una valoración de 9-10, al periodista tan solo el $13 \%$ valora como muy buena, siendo la última de las profesiones de las que preguntaba el Centro de Investigaciones Sociológicas (2013). En la misma encuesta se indica que la profesión de periodista está entre las peor valoradas, el 8,3\% la consideran como muy mal, junto a las relacionados con la justicia (juez $(12,4 \%)$, abogado $(8,4 \%)$ ) o policía local $(8,9 \%)$.

En esta investigación se pretende mostrar cuál es la situación laboral de los periodistas en España a partir del impacto de la pandemia provocada por la Covid-19 en 2020 y en qué situaciones a futuro pueden encontrarse en el futuro en unos pocos meses-años a partir de las proyecciones de desempleo en otras crisis.

\subsection{Impacto del Covid-19 en la sociedad}

La situación mundial a partir de la pandemia Covid-19 está generando situaciones de incertidumbre, inseguridades y reordenación de muchos de los valores sociales y económicos. En pocas semanas, un virus que aparecía en la ciudad China de Wuhan, se extendió por todo el planeta. Su rápida propagación, su transmisión y su gravedad obligaban a tomar medidas desconocidas hasta el momento: distancia social, higiene de manos, uso de mascarillas... pero con todo no fue suficiente y obligaba a los gobiernos a tomar decisiones más drásticas, como el cierre de todo lo considerado no esencial, establecer normativas para limitar los movimientos, obligando a permanecer en casa durante meses y a proceder a procesos de desescalada económica y social.

Meses después del inicio de la pandemia y a la espera de una vacunación masiva las limitaciones sociales y económicas se mantienen con diferente impacto a lo largo del planeta. En España, en marzo de 2020 se decretó el primer estado de alarma y confinamiento de la población. Las escuelas, institutos y universidades improvisaron clases virtuales y muchos trabajadores se acogieron al Expediente de Regulación Temporal de Empleo (ERTE). Las personas permanecieron por regla general en confinamiento domiciliario hasta mayo, exceptuando algunas actividades permitidas en el exterior como asistir al puesto de trabajo, ir al supermercado, sacar la basura, pasear al perro o realizar paseos en determinadas franjas horarias. A partir de mayo, se inició una desescalada en cuatro fases. En el mes de octubre, se 
aprobó el segundo estado de alarma con algunas medidas generales para todas las comunidades autónomas como la obligatoriedad de limitar la circulación de las personas por las vías o espacios de uso público - más conocido como toque de queda - entre las 23,00 y las 6,00 o, en su defecto, entre las 22,00 y las 0,00 horas y su fin entre las 05,00 y las 07,00 horas.

Volviendo a la escala mundial, la urgencia sanitaria está poniendo en evidencia el sistema económico fundamentado en las libertades empresariales del individuo, el derecho a la propiedad privada, mercados libres y libertad de comercio (Harvey, 2007). Este neoliberalismo genera desigualdad, pobreza (Pollin, 2013), influye en el cambio climático (Almiron, Boykoff, Narberhaus, y Heras, 2020) y descontentos (Prieto del Campo, 2013) y provoca un impacto sin precedentes en la salud mental de las personas.

La pandemia no ha finalizado y las dimensiones de salud siguen siendo muy preocupantes, así a marzo de 2021, el número de personas fallecidas casi alcanza los 2,6 millones y más de 117 millones de contagiados (European Centre for Disease Prevention and Control, 2021). Estos datos muestran la situación de la emergencia sanitaria y adelantan la situación laboral de millones de personas, la Organización Internacional del Trabajo (2020) prevé la pérdida de 245 millones de empleo y una pérdida de ingresos provenientes del trabajo por encima del $10 \%$ y alcanzando el $15,1 \%$ en países de ingreso mediano bajo.

\subsection{Impacto del Covid-19 en la sociedad}

El periodismo, como oficio, ha estado en primera línea de la pandemia desde el primer día, como la corresponsal de RTVE en China, Mavi Doñate, que aseguraba no tener miedo al contagio del coronavirus, pero sí respeto (Millán, 2020). Tenía motivos para el respeto, como ha reportado Press Emblem Campaign (2021) de Ginebra, 840 periodistas de 68 países han muerto a causa Covid-19. Más de la mitad en América Latina (458), seguido por Asia, con 151 muertes, en Europa, 147 muertes, en América del Norte 45 muertos y África, con 39 muertes. En España el número de periodistas muertos por Covid-19 alcanza los 12.

En España una de las primeras informaciones sobre el Covid-19 fue intervención del corresponsal de TVE en Milán, Lorenzo Milá, quien aseguraba el 25 de febrero de 2020 que parecía que "se extendía más el alarmismo que los datos" y explicaba que "estamos ante un nuevo tipo de gripe" que afecta "sobre todo a personas con las defensas bajas o situaciones de salud precarias". La intervención del corresponsal de la cadena pública fue muy aplaudida por diversos sectores de la población, entre ellos por profesionales de la información, académicos y asociaciones de prensa, que las comparaban con otras apariciones más alarmantes de reporteros en televisión con mascarillas y guantes.

Respecto al consumo de medios de comunicación, tal y como indica Casero-Ripollés (2020), durante el tiempo de emergencia sanitaria se produjo un aumento considerable del consumo informativo, que en televisión supuso un récord histórico durante los meses de abril y marzo de 2020 se incrementó $+40 \%$ y $37 \%$ respectivamente (Barlovento Comunicación, 2021). El uso de televisión ha registrado un promedio de 4 horas y 35 minutos por persona y día, un $16 \%$ más que el mismo periodo del año anterior (Tabla 1).

Los mayores incrementos de consumo televisivo se produjeron en todas las franjas del día siendo las franjas de mañana (de 07:00h a 14:00h) con un 21\% y la tarde (De 17:00h a 20:30h) 
con un $20 \%$ las que más crecieron, dejando la franja de prime time en crecimiento de $13 \%$ para alcanzar los 17,8 millones de audiencia (Barlovento comunicación, 2021).

Tabla 1

Evolución mensual minutos persona/día total consumo televisión 09/03/2020 a 09/03/2021

\begin{tabular}{|c|c|c|}
\hline Mes & Minutos persona/día en 2019 & Minutos persona/día en 2020-2021 \\
\hline Marzo 2020 & 249 & 341 \\
\hline Abril 2020 & 250 & 349 \\
\hline Mayo 2020 & 238 & 297 \\
\hline Junio 2020 & 230 & 255 \\
\hline Julio 2020 & 213 & 226 \\
\hline Agosto 2020 & 201 & 222 \\
\hline Septiembre 2020 & 229 & 242 \\
\hline Noviembre 2020 & 236 & 259 \\
\hline Diciembre 2020 & 254 & 279 \\
\hline Enero 2020 & 250 & 280 \\
\hline Febrero 2020 & 258 & 295 \\
\hline
\end{tabular}

De la misma forma, otros espacios de consumo de ocio televisivo han aumentado, como es el caso de las plataformas denominadas "on demand" (vídeo bajo demanda -VOD-) como Netflix, HBO o Prime Video con cifras de aumentos de audiencia millonarios durante la pandemia (Refojos, 2020). Y no solo por el ocio, también para la enseñanza y la formación han permitido un aumento en el consumo de Internet, aunque no con la suficiente calidad (Hodges, Moore, Lockee, Trust, y Bond, 2020).

Sin embargo, estos excelentes resultados de aumento de las audiencias han ido en dirección inversa. Se ha reducido el mercado global en España en más de 10\% con unas pérdidas ascendieron a más de 140 millones de euros, de ellos, los diarios perdieron 25 millones de euros, las radios 20 millones y la televisión dejó de ingresar 58,5 millones de euros (Casero-Ripollés, 2020).

\subsection{Incremento de noticias falsas e infodemia}

En el ecosistema de la posverdad indican Romero-Rodríguez, Valle y Torres-Toukoumidis (2018) se impone "la manipulación de creencias y emociones, de forma deliberada, con el fin de influir en el comportamiento social" (p. 263).

La pandemia y las decisiones en la emergencia sanitaria y socioeconómicas han sido un caldo de cultivo ideal para la aparición de bulos o desinformaciones, como indican Villa Gracia, y Cerdán Martínez (2020) que, a mitades de junio de 2020, se recopilan en España casi 600 bulos o desinformaciones, entre otros: la falta de evidencia de la cura a través del clorito de sodio, realizar gárgaras con vinagre, agua tibia o sal para prevenir el contagio o la prohibición de la 
OMS de la realización de autopsias. Estas noticias falsas se difunden especialmente en las redes sociales como Whatsapp, Twitter, Facebook Youtube Instagram, aunque también en medios periodísticos (Salaverría, Buslón, López-Pan, León, López-Goñi, y Erviti, 2020).

Villa y Cerdán (2020) apuntan a que durante la pandemia ha existido una tendencia correlativa entre el interés de los españoles por las noticias falsas y el incremento de muertes por coronavirus. Por ello, la OMS puso en marcha un equipo de comunicación de riesgo y gestión de infodemias para rastrear activamente la información, a la vez que habilitó en su web un apartado para desmentir mitos que circulaban por redes sociales (Aguado-Guadalupe y Bernaola-Serrano, 2020), y el Gobierno de España activó la campaña 'Coronavirus, siempre fuentes oficiales' (Martín-Barato et al., 2020).

Por otra parte, la opinión pública ha acuñado el término infodemia, vocablo empleado para referirse a la sobreabundancia de información (alguna rigurosa y otra falsa) sobre un tema. La Fundación del Español Urgente (Fundéu RAE, 2020) lo ha considerado válido y la Organización Mundial de la Salud (OMS) ha empleado también el anglicismo infodemic. El propio director general de la OMS aseguró que la evolución del brote de Covid-19 dependería de la medida en la que se hiciera llegar la información correcta a las personas que la necesitan (Ghebreyesus y $\mathrm{Ng}, 2020)$.

La necesidad de formación a la ciudadanía para hacer frente a la información falsa, a los bulos y a la posverdad es una medida eficaz (Blanco-Herrero y Arcila-Calderón, 2019) y, no solo ante estas informaciones falsas en las redes sociales, si no también, ante aquellas desinformaciones vertidas como parte de la estrategia comunicativa de grupos con claros intereses socioeconómicos (Rodrigo-Cano, 2020).

Por otro lado, un estudio sobre las portadas de Núñez-Gómez et alt (2020) informa de un "tono catastrofista" en las mismas, que hizo referencia fundamentalmente a la gestión del Gobierno, a la ansiedad, incertidumbre y estado de depresión psicológica y económica. Según los autores, los diarios más alejados ideológicamente del actual Gobierno presentaron los titulares más negativos.

Ante esta situación, es necesario conocer el estado del periodismo como profesión respecto al empleo, para ello, en esta investigación se pretende identificar esta situación tanto respecto al género como a las comunidades autónomas.

\section{METOdOLOGÍA}

Para conocer las repercusiones en el desempleo del periodismo de la pandemia provocada por la Covid-19 se ha realizado un análisis de los informes anuales de la profesión que realiza la Asociación de la Prensa de Madrid (APM) que publica anualmente la evolución del desempleo en el periodismo. Para ello se han descargado los datos del paro registrado desde 2008 hasta 2020 (Asociación de la Prensa de Madrid, 2009-2021) tanto por género como por comunidades autónomas.

\section{RESULTADOS}

La evolución del desempleo en el periodismo español desde la anterior gran crisis provocada por el estallido de la burbuja inmobiliaria en 2008 que generó el año negro para el desempleo en España superando los 3,5 millones de personas desempleadas en el periodo 2008-2012, con 
una tasa de paro del 25\% (Sanromá, 2012). En 2021, en plena pandemia, los datos de desempleo en España vuelven a alcanzar datos de 4 millones de parados y un 23,5\% de tasa (Sepe, 2021).

En esta misma línea ha ido aumentando el paro registrado de los periodistas y, en concreto, creció un 25\% en 2020 con respecto al mismo mes de 2019 (Asociación de la Prensa de Madrid, 2021). Estos datos son similares a los de 2011 en el que se alcanzó los 8520 parados en periodismo y las cifras en 2021 son de 8.536 (Tabla 2).

Tabla 2

Evolución mensual minutos persona/día total consumo televisión 09/03/2020 a 09/03/2021

\begin{tabular}{|l|l|l|l|}
\hline \multicolumn{1}{|c|}{ Año } & \multicolumn{1}{c|}{ Mujer } & \multicolumn{1}{c|}{ Hombre } & \multicolumn{1}{c|}{ TOTAL } \\
\hline 2008 & 2.768 & 1.778 & 4.546 \\
\hline 2009 & 4.373 & 2.722 & 7.095 \\
\hline 2010 & 4.945 & 2.722 & 7.667 \\
\hline 2011 & 5.492 & 3.028 & 8.520 \\
\hline 2012 & 6.652 & 3.807 & 10.459 \\
\hline 2013 & 6.661 & 3.899 & 10.560 \\
\hline 2014 & 6.029 & 3.422 & 9.451 \\
\hline 2015 & 5.547 & 3.133 & 8.680 \\
\hline 2016 & 5.029 & 2.861 & 7.890 \\
\hline 2017 & 4.558 & 2.579 & 7.137 \\
\hline 2018 & 4.323 & 2.505 & 6.828 \\
\hline 2019 & 4.409 & 2.594 & 7.003 \\
\hline 2020 & 5.290 & 3.246 & 8.536 \\
\hline
\end{tabular}

Lo preocupante es la tendencia, como se puede observar en la Figura 1, siguiendo el modelo de la anterior crisis lo peor está por llegar y se podría alcanzar cifras por encima de los 10.000 desempleados. En este análisis se parte de las semblanzas en la evolución del desempleo que tuvo lugar tras la crisis de 2008, en la que los datos de desempleo fueron en aumento desde 2008 hasta 2013. Si se repite la tendencia mostrada, los próximos 5 años pueden ser demoledores para el empleo de los periodistas.

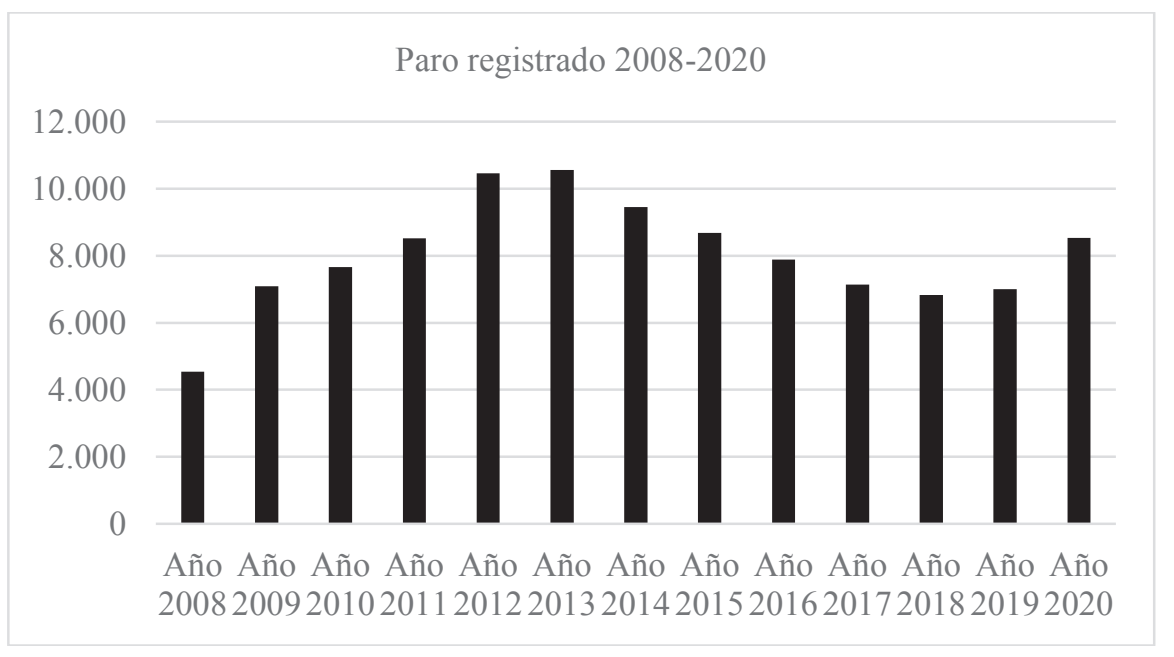

Figura 1. Paro registrado 2008-2020

Fuente: Asociación de la prensa de Madrid 2009-2021 
Otro de los datos preocupantes es el desempleo de la mujer periodista que se mantiene por encima del $60 \%$ con proyecciones cercanas al $65 \%$. Este dato es reiterado y la profesión de periodismo sigue siendo muy predominante entre las mujeres periodistas y que coincide con los mismos porcentajes de tituladas universitarias en periodismo (63\%) (Asociación de la Prensa de Madrid, 2021).

Por comunidades autónomas, Madrid, con 2.668 periodistas parados, Andalucía, con 1.406, y Catalunya, con 1.050, son las que encabezan el desempleo en la profesión (Tabla 3). De la misma forma, estas tres comunidades registran también aumentos por encima de la media en 2020. Se trata de las comunidades son también las más pobladas. Por el contrario, las que cuentan con menos desempleo son Ceuta y Melilla -15 parados-, La Rioja -37-, Navarra-106- y Extremadura -107-. El caso de La Rioja es destacable, además, por ser una de las pocas que el número de parados ha descendido de forma significativa (37\%), lo mismo ocurre con Islas Baleares (54\%).

Tabla 3

Paro registrado en periodismo y por comunidades autónomas 2008-2020

\begin{tabular}{|l|r|r|r|r|r|r|r|r|r|r|r|r|r|}
\hline & $\mathbf{2 0 0 8}$ & $\mathbf{2 0 0 9}$ & $\mathbf{2 0 1 0}$ & $\mathbf{2 0 1 1}$ & $\mathbf{2 0 1 2}$ & $\mathbf{2 0 1 3}$ & $\mathbf{2 0 1 4}$ & $\mathbf{2 0 1 5}$ & $\mathbf{2 0 1 6}$ & $\mathbf{2 0 1 7}$ & $\mathbf{2 0 1 8}$ & $\mathbf{2 0 1 9}$ & $\mathbf{2 0 2 0}$ \\
\hline Andalucía & 553 & $\mathbf{2 0}$ & $\mathbf{9 1 9}$ & $\mathbf{1 1 9 9}$ & $\mathbf{1 5 0 1}$ & $\mathbf{1 4 5 9}$ & $\mathbf{1 3 6 2}$ & $\mathbf{1 3 4 4}$ & 1280 & 1130 & 1045 & 1044 & 1406 \\
\hline Aragón & 56 & 101 & 108 & 145 & 165 & 181 & 145 & 143 & 135 & 96 & 97 & 122 & 160 \\
\hline Asturias & 44 & 123 & 142 & 153 & 221 & 215 & 181 & 154 & 137 & 128 & 127 & 118 & 159 \\
\hline Canarias & 128 & 218 & 224 & 258 & 323 & 311 & 287 & 274 & 227 & 215 & 204 & 231 & 184 \\
\hline Cantabria & 62 & 76 & 72 & 100 & 129 & 114 & 95 & 115 & 105 & 101 & 84 & 87 & 138 \\
\hline Castilla La Mancha & 74 & 169 & 179 & 272 & 355 & 336 & 291 & 247 & 201 & 216 & 203 & 230 & 251 \\
\hline Castilla y León & 179 & 255 & 303 & 345 & 452 & 500 & 409 & 405 & 349 & 290 & 310 & 300 & 360 \\
\hline Catalunya & 641 & 950 & 1000 & 1104 & 1348 & 1292 & 1058 & 920 & 870 & 721 & 792 & 845 & 1050 \\
\hline Ceuta y Melilla & 4 & 5 & 5 & 6 & 13 & 15 & 17 & 10 & 15 & 22 & 8 & 11 & 15 \\
\hline Comunidad Valenciana & 366 & 612 & 695 & 705 & 870 & 998 & 1020 & 918 & 770 & 633 & 562 & 622 & 693 \\
\hline Extremadura & 50 & 71 & 107 & 120 & 146 & 143 & 153 & 148 & 120 & 116 & 90 & 98 & 107 \\
\hline Galicia & 196 & 347 & 384 & 429 & 521 & 535 & 504 & 470 & 383 & 425 & 395 & 404 & 465 \\
\hline Islas Baleares & 87 & 94 & 132 & 178 & 213 & 179 & 131 & 120 & 104 & 89 & 81 & 81 & 179 \\
\hline La Rioja & 13 & 26 & 32 & 40 & 56 & 56 & 43 & 42 & 44 & 38 & 28 & 47 & 37 \\
\hline Madrid & 1638 & 2509 & 2596 & 2622 & 3206 & 3207 & 2853 & 2567 & 2446 & 2282 & 2219 & 2210 & 2668 \\
\hline Murcia & 58 & 112 & 128 & 169 & 182 & 223 & 178 & 154 & 136 & 137 & 122 & 144 & 173 \\
\hline Navarra & 74 & 120 & 148 & 148 & 172 & 183 & 173 & 154 & 152 & 125 & 109 & 96 & 106 \\
\hline País Vasco & 323 & 482 & 493 & 527 & 586 & 613 & 551 & 495 & 416 & 373 & 352 & 313 & 385 \\
\hline TOTAL & 4546 & 7095 & 7667 & 8520 & 10459 & 10560 & 9451 & 8680 & 7890 & 7137 & 6828 & 7003 & 8536 \\
\hline
\end{tabular}

\section{DISCUSIÓN Y CONCLUSIONES}

A pesar de la importancia que se le ha dado al periodismo tanto para la información como para hacer frente a las noticias faltas y a la reclamación ciudadana de la necesidad de más periodismo científico y local, la desinversión en publicidad de los años 2020-2021 pueden provocar datos de desempleo en el periodismo como en la anterior crisis en la que se superaron los 10.500 desempleados en periodismo. 
Además, puede tratarse de un perfil profesional a cubrir por los periodistas que, por un lado, contribuya a una democracia más saludable y, por otro, favorezca la generación de empleo en un sector especialmente afectado por la crisis económica.

Algunos de los desafíos a los que hace frente la profesión del periodista son la desinformación, el descrédito, la precariedad y el desempleo. En cuanto a la desinformación, todo ello sin obviar que puede ocurrir que la ciudadanía tome como noticia una información que no lo es y que, por tanto, persigue otros fines.

Además, es conveniente remarcar la tendencia de algunos políticos de tildar de noticia falsa una información que les pone en duda o desacredita. Se trata de una práctica que pone en tela de juicio la credibilidad de los profesionales de los medios de comunicación y que contribuye, aún más, a la mala reputación de la profesión.

Ante esta realidad, es necesario seguir investigando los bulos, las noticias falsas y la desinformación que permita a la ciudadanía mantener conductas reflexivas ante las comunicaciones tanto de los medios de comunicación como de las redes sociales (Salaverría et al, 2020) y especialmente en tiempos de emergencia sanitaria (Villa y Cerdán, 2020). Actualmente, es necesaria la creación de plataformas que destapen los bulos y las desinformaciones en las que haya periodistas especializados en datos, análisis y destaparlas. De la misma forma, estas plataformas pueden formar a más periodistas en la búsqueda de la verdad $y$, en general, a la ciudadanía de forma que exista un aprendizaje para descubrir las noticias falsas. En este sentido, convendría que las asociaciones de la prensa reforzaran la alfabetización mediática en las escuelas con el fin de que desde la infancia se adoptara una actitud crítica con los medios de comunicación. Según Domínguez (2010), poner al alumno en el rol de periodista lo estimula a leer, preguntar, analizar, ser críticos, y también a reforzar su capacidad comunicativa para divulgar los resultados de su opinión.

Por otra parte, la precariedad de los profesionales de la información influye en el contenido periodístico y se traduce, por tanto, en piezas con menos contexto, menos profundidad y más tendencia a la anécdota que a un análisis pormenorizado. En esta línea, la investigación de Suárez-Villegas et alt (2009), basada en encuestas a periodistas andaluces, afirmaba que muchos profesionales consideran que hay "graves problemas pues no se cubren las necesidades básicas de contrato, horario y salario". Los investigadores recogían que muchos encuestados apuntaron a que "al final se copia y pega" y, de este modo, "no sólo la calidad de la información se ve perjudicada por estas circunstancias laborales, sino que también la ética de los periodistas". En definitiva, un periodista precario es más dócil, tiene temor a perder su empleo y puede terminar por cambiar a empleos menos cualificados con los que obtiene mejores condiciones laborales y salariales.

\section{Referencias}

Aguado-Guadalupe, G., y Bernaola-Serrano, I. (2020). Verificación en la infodemia de la Covid-19. El caso Newtral. Revista Latina de Comunicación Social, 78, 289-308. https://doi.org/10.4185/RLCS$\underline{2020-1478}$

Almiron, N., Boykoff, M., Narberhaus, M., y Heras, F. (2020). Dominant counter-frames in influential climatecontrarian European think tanks. Climatic Change, 162:2003-2020. https://doi.org/10.1007/s10584-020-02820-4 
Asociación de la Prensa de Madrid (2009-2020). Informe anual de la profesión periodística. https://bit.ly/3bVUSZW

Barlovento Comunicación (2021, 11 de marzo). Balance del consumo de televisión durante el primer año del Covid. barloventocomunicacion.es. https://bit.ly/30W4y09

Blanco-Herrero, D., y Arcila-Calderón, C. (2019). Deontología y noticias falsas: estudio de las percepciones de periodistas españoles. Profesional De La Información, 28(3). https://doi.org/10.3145/epi.2019.may.08

Casero-Ripollés, A. (2020). La COVID-19 en el periodismo: un impacto ambivalente. RAEIC, Revista de la Asociación Española de Investigación de la Comunicación, 14 (7), 2-26. https://doi.org/10.24137/raeic.7.14.1

Centro de Investigaciones Sociológicas (2013). Estudio 2978. Barómetro de Febrero 2013. https://bit.ly/3c6s6G8

Domínguez Lázaro, M.d.I.R. (2010). Los medios en el aula: el periodismo como recurso pedagógico. En Una concienciación necesaria: I Jornadas Universitarias de Comunicación y personas con discapacidad, Universidad de Sevilla, Sevilla

European Centre for Disease Prevention and Control (2021). Situation update week 9, updated 11 March 2021. http://bit.ly/3109/sg

Fundéu RAE (2020, 24 de marzo). Infodemia, neologismo válido. fundeu.es. http://bit.ly/2OPDNIr

Ghebreyesus T.A., y Ng, A. (2020, 18 febrero). Desinformación frente a medicina: hagamos frente a la 'infodemia'. El País. http://bit.ly/2QbQTjt

Grecko, Témoris (2021, 5 de junio). Opinion: Los periodistas trabajan precarizados y expuestos al COVID19 en México. The Washington post. https://wapo.st/3vE7SLu

Harvey D. Breve historia del neoliberalismo. Madrid, Akal. 2007

Hodges, Ch. Moore, S. Lockee, B., Trust, T., y Bond, A. (2020). The Difference Between Emergency Remote Teaching and Online Learning. Educase Review. https://bit.ly/30VU5lz

Martín-Barato, A., López-Doblas, M., Luque-Martín, N., y March-Cerdá, J. C. (2020, abril 15). Fake news y bulos contra la seguridad y la salud durante la crisis del coronavirus. Escuela Andaluza de Salud Pública. https://bit.ly/3190Qkd

Millán, M. (2020, 13 febrero). Mavi Doñate: "No tengo miedo al contagio del coronavirus, pero sí respeto". Heraldo.es. https://bit.ly/3qVonzl

Núñez-Gómez, P.; Abuín-Vences, N.; Sierra-Sánchez, J. y Mañas-Viniegra, L. (2020). El enfoque de la prensa española durante la crisis del Covid-19. Un análisis del framing a través de las portadas de los principales diarios de tirada nacional. Revista Latina de Comunicación Social, 78,41-63.

Organización Internacional del Trabajo (2020). Observatorio de la OIT: La COVID-19 y el mundo del trabajo. Sexta edición. Estimaciones actualizadas y análisis. https://bit.ly/3vEogf1

Pollin, R. (2013). La lucha para derrotar al neoliberalismo global. La centralidad de las políticas sobre los mercados financieros en Ecuador y en otros lugares. En Pedro Chaves Giraldo, Carlos Prieto del Campo y René Ramírez Gallegos (eds.), Crisis del capitalismo neoliberal, poder constituyente y democracia real (pp. 149-174). Traficantes de sueño.

Press Emblem Campaign (2021, 4 marzo). In one year, at least 840 journalists have died from Covid-19. https://bit.ly/30UYyEV

Prieto del Campo, C. (2013). Capitalismo, poder constituyente e hipótesis comunistas para la transformación antisistémica. En Pedro Chaves Giraldo, Carlos Prieto del Campo y René Ramírez Gallegos (eds.), Crisis del capitalismo neoliberal, poder constituyente y democracia real (pp. 2358). Traficantes de sueño.

Refojos, M. (2020, 28 octubre). 2020, el año del streaming: así está cambiando el mundo esta tendencia. Elperiodico.com. https://bit.ly/31rH6l9 
Rodrigo-Cano, Daniel (2020). El factor R-Elacional para la educación ambiental. En José Antonio Gabelas y Carmen Marta-Lazo (Eds), La era TRIC: Factor R-elacional y Educomunicación. (pp. 242-244). Egregius: Zaragoza.

Romero-Rodríguez, L.M., Valle, A.L. y Torres-Toukoumidis, Á. (2018). Hacia una construcción conceptual de las Fakenews: Epistemología y tipologías de las nuevas formas de desinformación. En María José Pérez Serrano, Gema Alcolea-Díaz y Antonia Nogales-Bocio (Eds) Poder y medios en las sociedades del Siglo XXI. (pp. 259-273). Ed. Egregius: Sevilla.

Salaverría, R., Buslón, N., López-Pan, F., León, B., López-Goñi, I., y Erviti, M.-C. (2020). Desinformación en tiempos de pandemia: tipología de los bulos sobre la Covid-19. El profesional de la información, 29(3). 290-315. https://doi.org/10.3145/epi.2020.may.15

Sanromá, E. (2012). El mercado de trabajo español en la crisis económica (2008-2012): desempleo y reforma laboral. Revista de Estudios Empresariales, 2, 29-57

Sepe (2021). Datos nacionales de paro registrado. Febrero 2021. https://bit.ly/3vKlyn4

Soengas X., Rodríguez Vázquez, Al., Abuín Vences N. (2014): La situación profesional de los periodistas españoles: las repercusiones de la crisis en los medios. Revista Latina de Comunicación Social, 69, 104- 124. 10.4185/RLCS-2014-1003

Suárez-Villegas, J.C., Romero-Domínguez, L.R. y Almansa Martínez, A. (2009). El periodismo en el espejo. La profesión analizada por periodistas andaluces. Ámbitos. Revista Internacional de Comunicación, 18 (pp. 157-175). https://doi.org/10.12795/Ambitos.2009.i18.11

Unesco (2007) Model curricula for journalism education. Colección de la UNESCO sobre los estudios de Periodismo. https://bit.ly/3lqwpz6

Villa Gracía, A. D., y Cerdán Martínez, V. (2020). Bulos durante la pandemia del Covid-19 en España: un estudio a través de Google Trends. Revista Latina de Comunicación Social, 78,169-182. https://www.doi.org/10.4185/RLCS-2020-1473

\section{Semblanza de los autores}

Daniel Rodrigo-Cano es doctor en comunicación por la Universidad de Huelva. Docente de Periodismo y Comunicación en el Centro San Isidoro, adscrito a la Universidad Pablo de Olavide de Sevilla. Es miembro de la Asociación de Periodistas Ambientales.

Noelia Ruiz-Alba es doctoranda por la Universidad de Sevilla, miembro del Grupo de Investigación Estructura, Historia y Contenidos de la Comunicación (Grehcco) y del Laboratorio de Estudios en Comunicación (Ladecom). Es redactora en la delegación andaluza de la agencia de noticias Europa Press. 